\title{
MODEL ANALYSIS OF ADAPTIVE CAR DRIVING BEHAVIOR
}

\author{
P.H. WEWERINKE \\ Department of Applied Mathematics \\ University of Twente \\ P.O. Box 217, 7500 AE Enschede \\ The Netherlands
}

\begin{abstract}
This paper deals with two modeling approaches to car driving. The first one is a system theoretic approach to describe adaptive human driving behavior. The second approach utilizes neural networks.

As an illustrative example the overtaking task is considered and modeled in system theoretic terms. Model results are used to teach a neural network. The results show that a neural network is able to learn this task even when certain task variables change.

The next step is to perform an experiment with real human operators in order to assess the validity of both modeling approaches and their relative merit.
\end{abstract}

\section{Introduction}

Man-machine systems in general and car driving specifically often involve time-varying and adaptive characteristics related to the system, the environment and/or time-varying human operator (HO) behavior. The resulting adaptive $\mathrm{HO}$ behavior is the topic of this paper.

One approach to describe adaptive man-machine systems is based on neural networks (NN), as an inputoutput model of the HO. Based on data(experience) a desired (given) input-output relationship can be learned by a NN. This approach will be considered in this paper. This research is part of an ongoing research project in which adaptive HO behavior is investigated and modeling approaches are compared based on system theory and NN. Although the aim of this research is to assess the relative merit of both model approaches to describe adaptive human behavior in operating dynamic systems in general, car driving is considered as a specific application. This allows a concrete task analysis and results.

In Chapter 2 car driving is analyzed and a model of the overtaking task is discussed. In this model it is assumed that the $\mathrm{HO}$ has learned the quantitative relationships between system- and task variables. However, in the case of inexperienced drivers and/or traffic developments (all) these relationships are not known precisely and the HO has to learn them. This adaptive behavior is discussed in Chapter 3 and the two approaches to model this behavior.

In Chapter 4 the overtaking task is simulated. Firstly the model of Chapter 2 was implemented in the MAT$\mathrm{LAB}$ program to generate overtaking results in terms of the three driving modes: waiting (following the preceding car at a given distance), go ahead (driving at a desired speed) and overtaking (accelerating in the right lane and overtaking in the left lane). Next the model results were used to teach a NN, to see whether a NN could reproduce the model results. Several task variables were varied to test the generality of the model results. Finally, Chapter 5 contains the conclusions.

\section{Model analysis of car driving}

\section{$2.1 \quad$ General}

Car driving consists of a number of primary and secundary tasks, which result from the overall goal to go from A to B. Primary tasks are of direct importance for a safe transport from A to B. Examples are lane keeping, speed control, car following, overtaking and navigation. Secundary tasks can support primary tasks or can be unrelated to them. Examples are handling a navigation system, a radio and a carphone.

In general the driver has to divide his attention among the various tasks to be performed. This attention allocation is modeled in [3]. Basically the strategy is to perform the most important task first, so as to achieve the overall goal.

In previous publications ([1] and [2]) models of lane keeping, car following and overtaking have been discussed as the main primary tasks. In addition in [1] these models are combined with a traffic flow model in order to obtain a model structure that describes the relationship between detailed traffic factors (related to driver behavior, traffic system design, environment, etc.) and overall measures of road capacity and safety.

The emphasis of this paper is on adaptive human op-

$0-7803-3280-6 / 96 / \$ 5.00 \mathcal{C}_{1996 \text { IEEE }} \quad-2558-$ 
erator behavior. This is a continuation of [2]. The car driving task although of interest as such, is used as an illustrative example. Because of this emphasis only the most complex driving task, the overtaking task, will be considered in this paper. For lane keeping, etc. the reader is referred to [1] and [2].

\subsection{Overtaking}

Overtaking is the most complex driving subtask including observing, information processing, decision making, planning, maneuvering and other traffic. The overtaking situation is shown in Fig. 1 for a two-lane road. The analysis for freeways is similar.

Assume the situation that car $i$ intends to pass the preceding car $j$ at distance $X_{j}$ ahead. The possibility to do so depends on the oncoming cars $k$ and $\ell$. At time $t_{0}=0$ car $i$ decides at distance $X_{j}$ from car $j$ to accelerate from speed $u_{j}$ to speed $u_{m}$, with time constant $T_{u}$. The distances to cars $k$ and $\ell$ (with speed $u_{\ell}$ ) are $X_{k}$ and $X_{\ell}$, respectively. At time $t_{1}$ car $i$ changes to the left lane. At time $t_{2}$ car $j$ has been overtaken and car $i$ returns to the right lane again. The two conditions which must be satisfied are that at $t_{1}$ car $k$ must be passed and car $\ell$ must be away far enough to allow the overtaking maneuver. This implies the two inequalities

$X_{k}<X_{j}+\left(u_{\ell}+u_{j}\right) t_{1}-S_{k}$

$X_{\ell}>X_{j}+S_{j}+S_{\ell}+\left(u_{\ell}+u_{j}\right) t_{2}$

with $S_{j}$ the distance which car $i$ is overtaking in the left lane with respect to car $j$ and $S_{k}$ and $S_{\ell}$ the safety distances to car $k$ and $\ell$, respectively.

Useful approximations can be derived [4] for $t_{1}$ and $t_{2}$ (for $t_{1}$ and $t_{2}$ smaller than $T_{u}$ ). The result is

$t_{1} \approx \sqrt{\frac{2.7 X_{j} T_{u}}{u_{m}-u_{j}}}$ and $t_{2} \approx \sqrt{\frac{2.7\left(X_{j}+S_{j}\right) T_{u}}{u_{m}-u_{j}}}$

Combining eq. (3) with (1) and (2) shows that the decision criteria (1) and (2) depend on $X_{j}$ (which can be identified with the overtaking strategy), car dynamics $\left(T_{u}\right)$, speeds and safety margins.

The required gap between car $k$ and car $\ell\left(X_{\ell}-X_{k}\right)$ can be determined as a function of $X_{j}$. It turns out that the required gap can be substantially reduced already for a small $X_{j}$. So the possibility to trade-off $X_{\ell}-X_{k}$ allows car $i$ to optimize its overtaking strategy depending on the momentaneous traffic situation.
The speed of car $i$ is represented by a first order process driven by a commanded speed $\left(u_{m}\right)$ and a first order disturbance input $\left(w_{u}\right)$. Other traffic is modeled with a constant average speed. The specific speed of each car is unknown to car $i$ and has to be estimated based on the observed (derivative of) distances to the other cars. The system model of the total process can be expressed in the general form

$X(k+1)=f(X(k), U(k), W(k))$

with $X=\operatorname{col}\left(w_{u}, u_{i}, X_{j}, X_{k}, X_{\ell}, X_{i k}, X_{i \ell}\right)$ with $X_{i k}$ and $X_{i \ell}$ the adjoint states representing the inequalities (1) and (2), combined with (3).

The distances to other cars can be estimated based on the outside visual cues. The nonlinear relationships can be expressed in the general form

$Y_{p}(k)=g(X(k-i))+v(k-i)$

with $v$ the observation noise and $i$ the time delay. By means of an extended Kalman filter the states can be estimated. The estimated variables $X_{i k}$ and $X_{i \ell}$ are used to decide whether or not overtaking is possible, in case the distance to the slower preceding car becomes smaller than a criterion value $X_{d}$. This involves a sequential decision process to determine continuously the mode of car $i$ : 1. car following with speed $u_{j}, 2$. driving with speed $u_{m}$ and 3 . overtaking, which is basically a pre-programmed maneuver (accelerating at $t_{0}$ till $u_{m}$ and lane changes at $t_{1}$ and $t_{2}$ ). This is summarized in Fig. 2 showing the decisions and corresponding modes involved in the driving task. In the following model analysis simulation results will be considered in terms of these three modes.

\section{Adaptive driver behavior}

Adaptive driver behavior can be related to the level of experience and to the adaptive human capability to traffic system developments. This can be related to

- the knowledge of the car response to control inputs (internal model of the vehicle dynamics);

- The use of the visual cues (accuracy, attention allocation);

- control strategy;

- the relationships between task variables, on which overtaking decisions and strategy, and time coordination are based. 
Conceptually, the HO builds up the knowledge of the task resulting in more and more efficient functioning, based on experience (data).

There are two obvious approaches to model such a learning process. The first is a system theoretic approach and the second one is based on the use of neural networks.

\subsection{System theoretic approach}

The system theoretic approach is based on the system model of equations (4) and (5). One extreme is that the HO knows the task completely based on the perfect knowledge of this model. Generally it can be assumed that the model is partly known (the functions $f$ and $g$ ). This is implemented in terms of a number of unknown parameters $\theta$ in the model given by

$X(k+1)=f(\theta(k), X(k), U(k), W(k))$

$Y_{p}(k)=g(\theta(k-i), X(k-i))+v(k-i)$

Learning is now modelled as a parameter estimation problem. The procedure to solve this is by adding the unknown parameter to the state vector using the parameter model $\theta(k+1)=\theta(k)$. The result is an augmented nonlinear system, again in standard form

$\tilde{X}(k+1)=\tilde{f}(\tilde{X}(k), U(k), W(k))$

$Y_{p}(k)=g(\tilde{X}(k-i))+v(k-i)$

with $\tilde{X}=\operatorname{col}(X, \theta) \quad \tilde{f}=\operatorname{col}(f, \theta)$. This can be solved by means of an extended Kalman filter to estimate $\tilde{X}$ and thus $X$ and $\theta$, based on new data $Y_{p}$ (experience). Prior knowledge of a naive $\mathrm{HO}$ has to be translated into the initial estimate $\hat{\tilde{X}}(0)$. This is a nontrivial problem. One possibility is to assume $\theta(0)=0$.

\subsection{Neural network approach}

Human operator behavior can be described as the relationship between task inputs $Y_{p}$ and control outputs $U$ (inputs to the system). Learning this functional relationship between $Y_{p}$ and $U$ can be described by a neural network $(\mathrm{NN})$.

Basically a NN consists of a number of processing elements (or neurons) with weighted connections [5]. The weights represent the memory of the network and determine the input-output relationship. The $\mathrm{NN}$ has a given structure, determined by the number of neuron layers, the number of neurons per layer and the connections between the neurons. In this study a commonly used structure is assumed with one input layer, determined by the inputs $Y_{p}$, one output layer, determined by the outputs $U$ and one hidden layer with 10 neurons. Only feedforward connections are assumed. Such a structure is known [6] to be able to approximate any arbitrary input-output relationship (function), in case enough hidden layer neurons are assumed.

Learning the NN, i.e. the scheme to adjust the weights, can be based on an explicit performance measure, e.g. to obtain a desired output. This is called supervised learning. A common learning strategy is the so-called backpropagation. This is also assumed in this study. Another learning strategy is called unsupervised learning, in which the adaptation scheme is not depending on the NN output.

The backpropagation algorithm can be considered as a first onder gradient (steepest descent) method. It has been shown to provide good optimization results in many applications with favourable computational simplicity.

For the car driving (overtaking) task the HO inputs $Y_{p}$ consist of speed and relative distances to preceding and oncoming cars. The outputs $U$ consist of gas and brakes.

\section{Simulation of the overtaking task}

The overtaking task including the system model as described in Chapter 2 was simulated. It was assumed that all cars have a constant speed of $20 \mathrm{~m} / \mathrm{s}$ and that car $i$ tries to drive $30 \mathrm{~m} / \mathrm{s}\left(u_{m}\right)$. The actual average speed, in the following expressed as the velocity $v_{r}$ above the $20 \mathrm{~m} / \mathrm{s}$, is the key overtaking performance measure. The experimental (independent) variables considered are the traffic densities in the right and left lane $\left(d_{r}\right.$ and $d_{\ell}$, in average number of cars per meter with a Poisson distribution), type of car, in terms of the time constant $T_{u}$ and overtaking strategy, in terms of the parameter $X_{d}$ (distance to the preceding car at the moment of accelerating).

The overtaking process is described in terms of the following phases (modes): mode 1: car $i$ has to wait behind car $j$ before overtaking is possible; mode 2: car $i$ can accelerate because the preceding car $j$ is away far enough; mode 3: car $i$ can overtake and accelerate in the right lane; mode 4: car $i$ overtakes and accelerates in the left lane; mode 5: car $i$ decelerates nominally be- 
cause of preceding car $j$; mode 6 ; car $i$ breaks to avoid getting too close to car $j$. To simplify the picture, modes 1,5 and 6 were pooled in one waiting mode 1 . Also mode 3 and mode 4 were pooled in one overtaking mode 3 .

The model results are shown in Fig. 3 for a simulation run of 5 minutes for the nominal condition $\left(d=0.01 / \mathrm{s}, d_{\ell}=0.005 / \mathrm{s}, T_{u}=10 \mathrm{~s}\right.$ and $X_{d}=10 \mathrm{~m}$, in addition to reasonable values for safety margins and decelerating). Because of the low traffic density in the left lane 15 overtakings were obtained (mode $3=15$ ). The NN results will be discussed in the following.

Next the NN was trained to learn the overtaking task. For this purpose the NN utilized the training data set of $300 \mathrm{~s}$, shown in Fig. 3. The inputs were the distances to the other cars $\left(X_{j}, X_{k}\right.$ and $\left.X_{\ell}\right)$ and the driving speed of car $i\left(u_{i}\right)$. The outputs were the (3) driving modes. 5000 iterations were made to obtain the NN results. These results are also shown in Fig. 3. Theoretically the $\mathrm{NN}$ should be able to duplicate the model results perfectly, depending on the initial weights, the number of neurons assumed and the number of iterations. However, an inspection of Fig. 3 shows that the most important chracteristics are reproduced.

One way to analyse the NN results more precisely is to define the NN output below 1.5 as mode 1 , above 2.5 as mode 3 and otherwise as mode 2. The results are shown in Fig. 4. It will be clear that by far most of the time the system model mode coincides with the NN mode. It is computed that this is $94 \%$ of the time. The same percentage pertains to the overtaking mode (3), which is the crucial mode as far as traffic safety is concerned. The overtaking maneuver is a pre-programmed maneuver determined by the corresponding initial and final time. As can be seen in Fig. 3 any difference in this mode is in the beginning (when accelerating in the right lane). The final time is duplicated precisely. This is important because it determines the end of the overtaking maneuver in the left lane before running into oncoming traffic.

In principle, it should be possible to improve the $\mathrm{NN}$ results by changing the number of neurons, the initial weights and/or by increasing the number of iterations. However, the results agree well enough to conclude that the NN is able to duplicate the model results.

After training the $\mathrm{NN}$ on the training data, a test set of data of $300 \mathrm{~s}$ was used to check how well the NN has learned the task. The result is given in Fig. 5, showing the same good results as before, especially, again, with respect to the last part of the overtaking maneuver.
The next question is how general the $\mathrm{NN}$ results are. In other words, suppose the tasks variables are changing, is the $N N$ driver still adequate?

The first variable considered was the car dynamics. The results of a faster car $\left(T_{u}=5 \mathrm{~s}\right.$ instead of $\left.10 \mathrm{~s}\right)$ is shown in Fig. 6. As can be seen the agreement between the system model and the $\mathrm{NN}$ is somewhat worse. Now, in $80 \%$ of the time the modes coincides. So, the $N N$ driver would require again some more practise to improve its performance. This is similar to the case of a human driver.

The next variable considered was the traffic density in the left lane. The traffic density was increased from $200 \mathrm{~m}$ to $175 \mathrm{~m}$ (average car distance). The $\mathrm{NN}$ driver of Fig. 3 and Fig. 5 was tested in this situation. The result is shown in Fig. 7. Only 5 overtakings were possible. About $75 \%$ of the time the driver was in the waiting mode (1). As can be seen from the figure the NN driver has problems with the waiting mode. Apparently, the learning of this mode (see figure 3 ) was not enough to deal with a situation in which waiting was the predominant mode. This makes sense, the more when one reconsiders the waiting mode results of Fig. 3 , seeing that the $\mathrm{NN}$ results are rather scattered.

The next step was to teach the $\mathrm{NN}$ for the larger traffic density case to see how the results could be improved. The result is shown in Fig. 8. Now, the waiting mode is clearly distinguished from the other modes (although still scattered; this might be removed by assuming less neurons in the hidden layer, or by a different - i.e. more nonlinear - activation function). The test of this result for the nominal traffic situation is shown in Fig. 9 . The agreement between the system model results and the NN results is good and comparable to the original results of Fig. 5. In other words, training the $\mathrm{NN}$ in the situation with larger traffic density is adequate to drive in the nominal traffic situation, but not the other way around as the waiting mode is the most difficult to learn as we have seen before.

\section{Concluding remarks}

In this paper adaptive car driving behavior is investigated. As an illustrative example the overtaking task is considered. A model of this complex task is discussed in system theoretical terms.

Learning this task can be modeled by means of an extended Kalman filter or in terms of a neural network.

The model simulation results of the overtaking task have been used to teach a neural network. The results 
show that a neural network is able to learn this task (duplicate the model results) even when certain task variables change (car dynamics and traffic density).

The next step is to perform an experiment with real human operators performing the overtaking task. The key independent variable is the experience level. The experimental results will be compared with the system theoretic (extended Kalman filter) results an with the neural network results in order to assess the validity of both modeling approaches and their relative merit to describe human learning behavior in car driving specifically and in operating dynamic systems in general.

\section{References}

[1] P.H. Wewerinke, "Modelling car driving and road traffic", Proc. IFAC MMS '95, Cambridge, U.S.A.

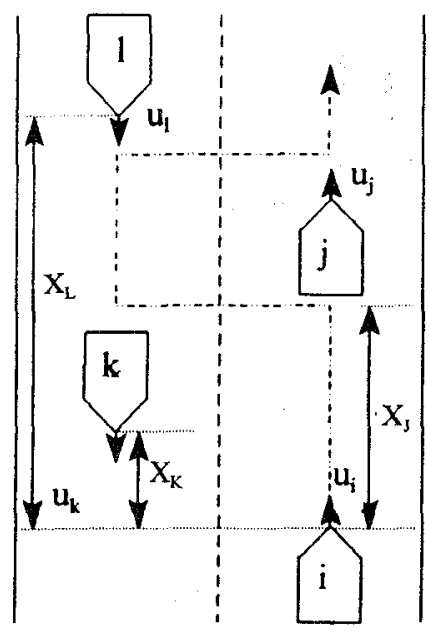

Fig. 1. Overtaking task situation

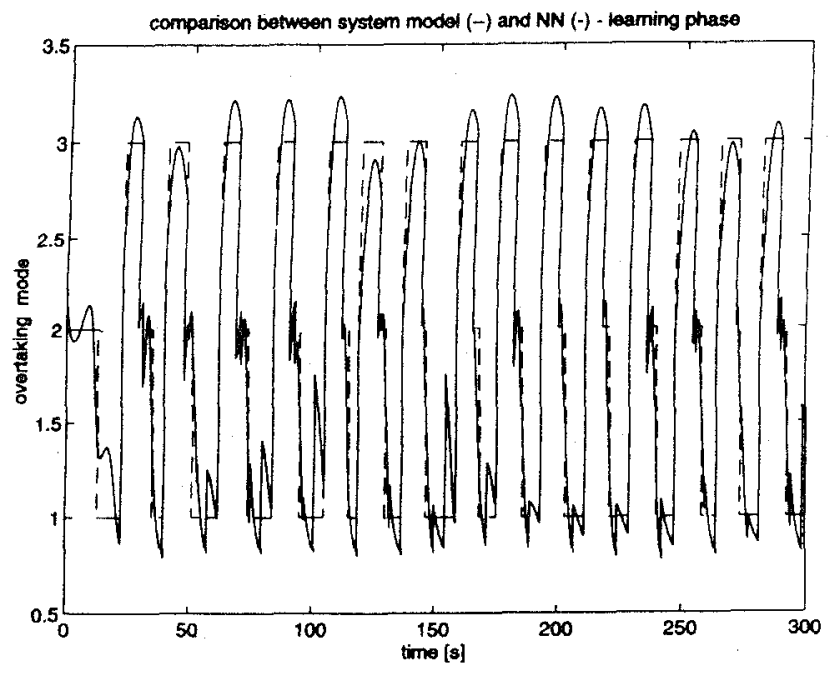

Fig. 3. Simulation results of the overtaking task in terms of the driving mode
[2] P.H. Wewerinke and H. Hilberink, "A comparison between system theoretic models and neural networks applied to car driving", Proc. 14th European annual conference on human decision making and manual control, Delft, 1995.

[3] P.H. Wewerinke, "Models of the human observer and controller of a dynamic systems", Ph.D. thesis, University of Twente, 1989.

[4] H. Hilberink, "A comparison of system theoretic models and neural networks applied to human learning involved in car driving", Thesis (in Dutch), Univ. of Twente, 1994.

[5] P.K. Simpson, "Artificial neural systems", Pergamon Press, 1990.

[6] W.T.C. van Luenen, "Neural networks for control on knowledge representation and learning", Ph.D. thesis, University of Twente, 1993.

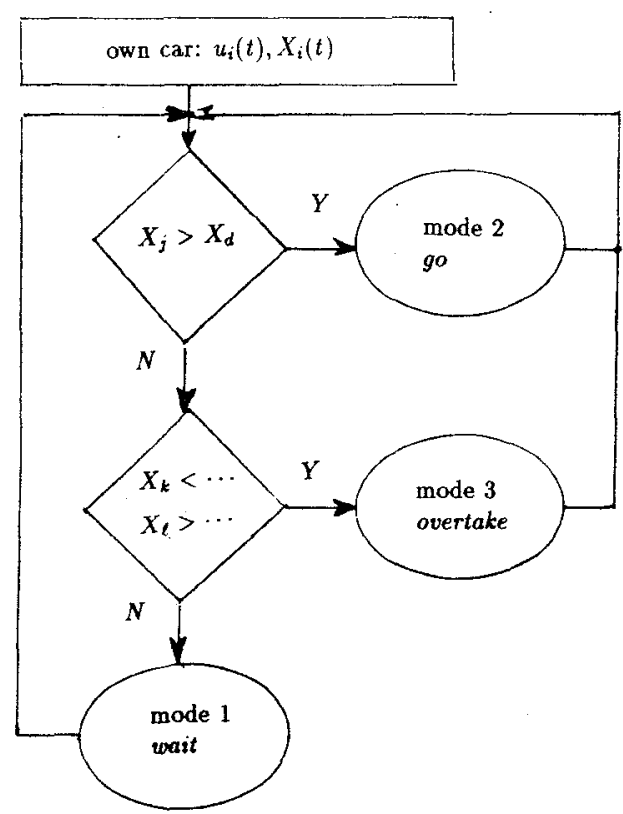

Fig. 2. Overtaking decisions and modes 


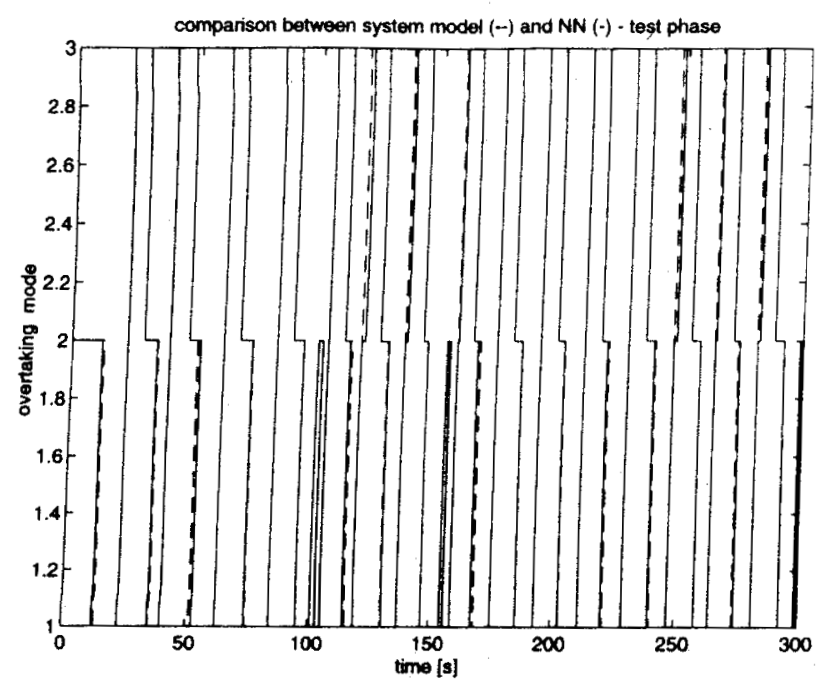

Fig. 4. System model and NN modes of the overtaking task

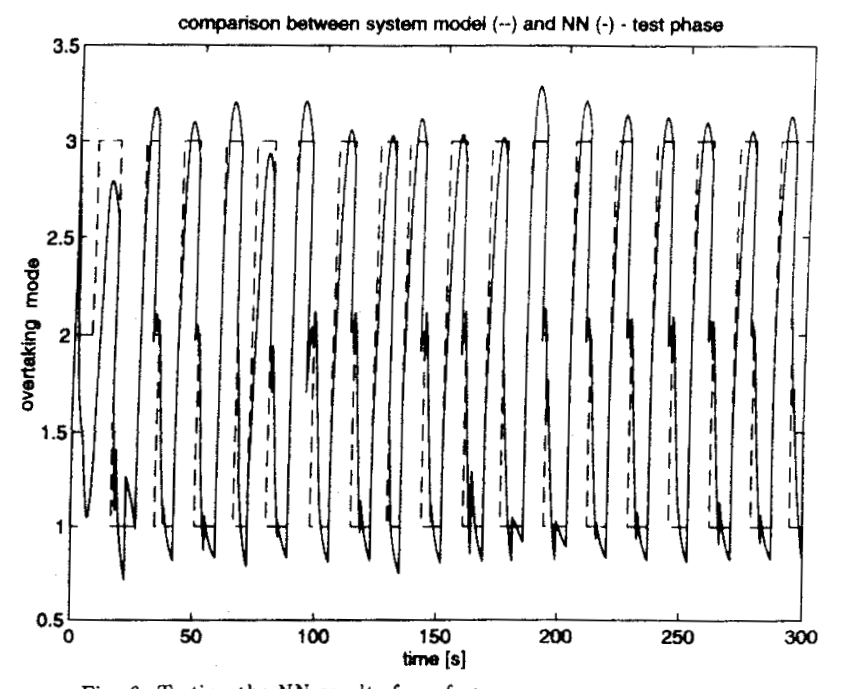

Fig. 6. Testing the $\mathrm{NN}$ results for a fast car

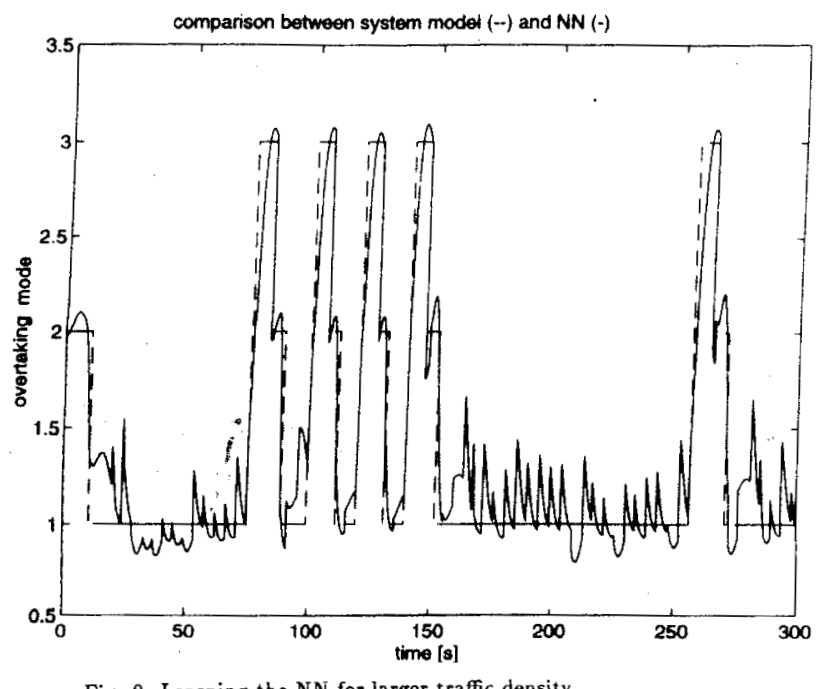

Fig. 8. Learning the NN for larger traffic density

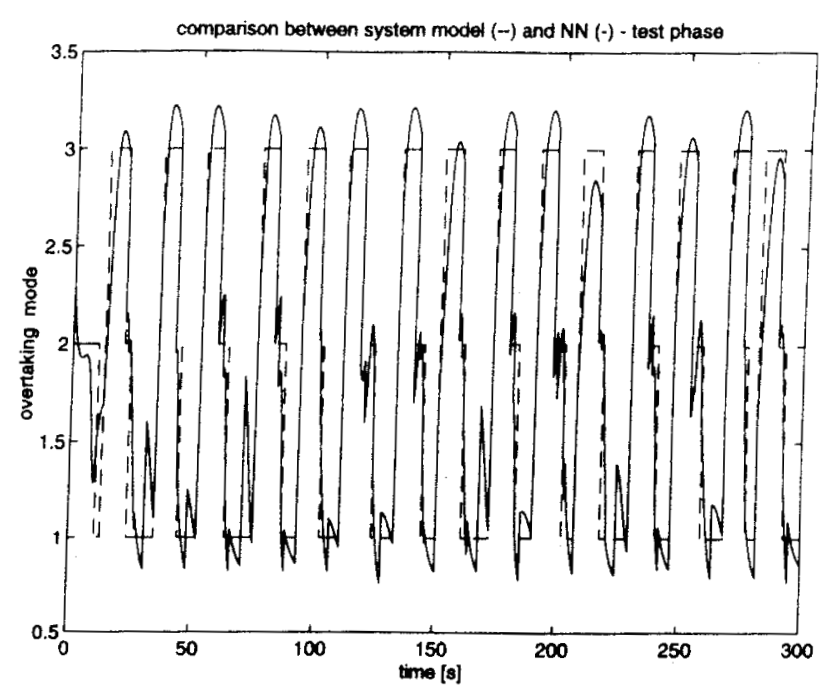

Fig. 5. Testing the NN results for the overtaking task

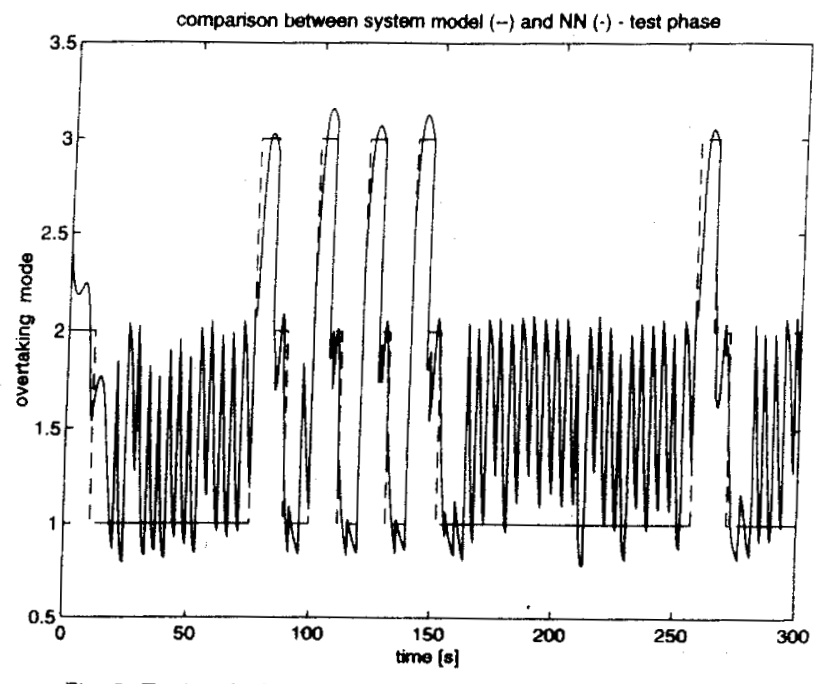

Fig. 7. Testing the NN results for larger trafic density

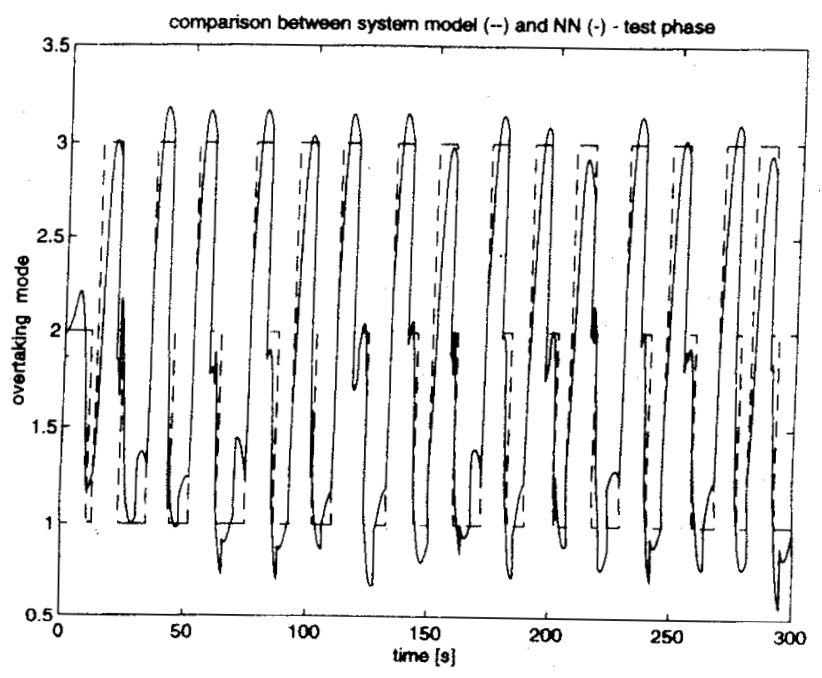

Fig. 9. Testing the NN results for the nominal traffic density situation 